\title{
Alexithymia, Suicide Ideation and Homocysteine Levels in Drug Naïve Patients with Major Depression: A Study in the "Real World" Clinical Practice
}

\author{
Domenico De Berardis ${ }^{1,2}$, Luigi Olivieri ${ }^{1}$, Gabriella Rapini ${ }^{1}$, Serena Di Natale ${ }^{1}$, Nicola Serroni ${ }^{1}$, \\ Michele Fornaro $^{3,4}$, Laura Orsolini ${ }^{4,5}$, Alessandro Valchera ${ }^{4,6}$, Alessandro Carano ${ }^{7}$, Federica Vellante ${ }^{1,2}$, \\ Paola Annunziata Varasano ${ }^{8}$, Gabriella Lucidi Pressanti ${ }^{8}$, Gianluca Serafini ${ }^{9}$, Maurizio Pompili ${ }^{10}$, \\ Giovanni Martinotti ${ }^{2}$, Massimo Di Giannantonio ${ }^{2}$

\begin{abstract}
'National Health Service, Department of Mental Health, Psychiatric Service of Diagnosis and Treatment, Hospital "G. Mazzini", Teramo, 2Department of Neurosciences and Imaging, University "G. D'Annunzio", Chieti, 'Department of Psychiatry, Federico II University, Naples, ${ }^{4}$ Polyedra, Teramo, Italy, ${ }^{5}$ School of Life and Medical Sciences, University of Hertfordshire, Hatfield, UK, 'Villa S. Giuseppe Hospital, Hermanas Hospitalarias, Ascoli Piceno, "Department of Mental Health, Psychiatric Service of Diagnosis and Treatment, Hospital "Madonna Del Soccorso", National Health Service, San Benedetto del Tronto, ${ }^{8}$ Department of Immunohematology and Transfusional Medicine, "G. Mazzini" Hospital, Teramo, ${ }^{9}$ Department of Neuroscience, Rehabilitation, Ophthalmology, Genetics, Maternal and Child Health, Section of Psychiatry, University of Genoa, Genoa, ${ }^{10}$ Department of Neurosciences, Mental Health and Sensory Organs, Suicide Prevention Center, Sant'Andrea Hospital, Sapienza University of Rome, Rome, Italy
\end{abstract}

\begin{abstract}
Objective: This study was performed to elucidate relationships between alexithymia, suicide ideation and homocysteine levels in drug-naïve outpatients with major depressive disorder (MDD).

Methods: Sixty seven outpatients with MDD with melancholic features were evaluated by the means of the Hamilton Depression Rating Scale, the Toronto Alexithymia Scale (TAS-20), the Scale of Suicide Ideation, and homocysteine levels.

Results: Alexithymic subjects showed higher scores on all scales and higher homocysteine levels. Regression analysis shown higher homocysteine levels and TAS-20" "Difficulty in Describing Feelings" dimension, in turn being associated with higher suicide ideation.

Conclusion: In conclusion, alexithymic MDD outpatients may characterize for homocysteine dysregulation that may be linked to suicide ideation, regardless depression' severity. However, study limitations are discussed and must be considered.
\end{abstract}

KEY WORDS: Affective symptoms; Alexithymia; Depression; Suicidal ideation; Homocysteine.

\section{INTRODUCTION}

Major depressive disorder (MDD) is one of the most disabling and common psychiatric disorders worldwide. ${ }^{1)}$ The relationship between MDD and alexithymia has been

Received: February 28, 2018 / Revised: May 6, 2018

Accepted: June 27, 2018

Address for correspondence: Domenico De Berardis, MD, PhD National Health Service, Department of Mental Health, Psychiatric Service of Diagnosis and Treatment, Hospital "G. Mazzini”, p.zza Italia 1, 64100 Teramo, Italy

Tel: +39-0861429708, Fax: +39-0861429709

E-mail: domenico.deberardis@aslteramo.it; dodebera@aliceposta.it ORCID: https://orcid.org/0000-0003-4415-5058 investigated in several studies. ${ }^{2,3)}$ Whenever associated, alexithymia is a risk factor for MDD itself, and/or a relatively stable personality trait further increasing the overall burden. ${ }^{4)}$

Homocysteine levels have been extensively investigated in MDD cases, especially in the elderly. ${ }^{5,6)}$ Higher homocysteine levels are often observed in course of MDD vs. control subjects and may represent a risk factor for poorer memory function and global cognitive performance, regardless depression severity. ${ }^{6)}$ However, to date, no studies have specifically assessed the relationships between alexithymia, suicide ideation and homocysteine

(c) This is an Open-Access article distributed under the terms of the Creative Commons Attribution Non-Commercial License (http://creativecommons.org/licenses/by-nc/4.0) which permits unrestricted non-commercial use, distribution, and reproduction in any medium, provided the original work is properly cited. 
levels.

To the best of our knowledge, this is the first study aiming at: i) testing whether any differential profile in homocysteine levels and suicidal ideation in MDD patients would depend on concurrent presence of alexithymia or not; ii) assessing the impact of selected clinical variables in the prediction of suicidal ideation using a blockwise linear regression analysis.

\section{METHODS}

A total of 67 adult outpatients (34 males, 33 females) with a Diagnostic and Statistical Manual for Mental Disorders, 4th edition, text revision (DSM-IV-TR) ${ }^{7}$ diagnosis of MDD with melancholic features were recruited at several mental health facilities across Central Italy in an everyday clinical practice setting. All diagnoses were made by psychiatrists with at least 5 years clinical experience and confirmed with the Structured Clinical Interview for DSM-IV (SCID) ${ }^{8}{ }^{8}$ Eligible patients met the criteria for a major depressive episode (MDE) with a score $\geq 16$ at the Hamilton Depression Rating Scale (HAM-D), 17-item version. ${ }^{9)}$ Patients' age ranged between 18 to 45 years, being naïve treatment seeker for MDE. Within subjects diagnosed with MDD we distinguished between patients with their first depressive episode $(n=47,70.1 \%)$ and patients with recurrent depressive episodes with one depressive episode before the present one $(n=20,29.9 \%)$, but never treated with antidepressants or other psychotropics. Thriry-nine patients (58.2\%) were smokers.

Patients receiving psychotherapy treatment were excluded. Additional exclusion criteria were the following; concurrent treatment with vitamin B supplements, any additional axis-I disorder, including substance use disorder, mental retardation or presence of any organic mental disorders.

Alexithymia was evaluated by the means of the Italian version $^{10)}$ of the 20-item Toronto Alexithymia Scale (TAS-20); a TAS-20 score $\geq 61$ was considered indicative of alexithymia. ${ }^{11)}$ The TAS-20 has a three-factor structure: Factor I assesses the ability to identify feelings and to distinguish between the feelings and bodily sensations of emotional arousal (difficulty in identifying feelings, DIF); Factor 2 reflects the inability to communicate feelings to other people (difficulty in describing feelings, DDF); Factor 3 assesses externally-oriented thinking (EOT).
In order to assess suicidal ideation, the total score of the clinician-rated Scale of Suicide Ideation (SSI) was administered. ${ }^{12)}$ MDD severity was assessed with the 17-item HAM-D total score. ${ }^{9)}$

Weight was measured (in light indoor clothing with shoes removed) using a balance beam scale, and height was measured using a stadiometer. Weight and height were used to calculate body mass index (BMI) expressed as $\mathrm{kg} / \mathrm{m}^{2}$. The mean $\mathrm{BMl}$ was $22.1 \pm 1.7 \mathrm{~kg} / \mathrm{m}^{2}$.

Serum homocysteine was measured collecting venous blood samples. Blood was drawn into tubes containing ethylenediaminetetraacetic acid/K3, placed on ice immediately thereafter, and centrifuged at $4^{\circ} \mathrm{C}$. Plasma was separated and immediately stored at $-80^{\circ} \mathrm{C}$ before it was analysed. Blood samples were collected between 7:00 am and 8:30 am, after the patient had fasted for at least 10 hours.

The rating scales' records and homocysteine measurements were collected as a part of everyday "real world", routine clinical practice evaluation and assessment of patients. However, each patient had to understand the nature of the study and signed a valid informed consent document prior to enrolment. A waiver was granted from institutional ethical committee as this was a retrospective study.

\section{Statistical Analysis}

The differences between individuals with and without alexithymia were tested using analyses of covariance (ANCOVA) with TAS-20 positivity/negativity as a factor and age, gender, smoking status, duration of illness, and recurrence and BMI scores as covariates. Cohen's $d$ effects sizes were likewise calculated. A block-wise linear regression analyses were performed to ascertain which variables were associated with the severity of suicidal ideation (SSI as dependent variable). Age, gender, smoking status, duration of illness, recurrence, BMI and HAM-D scores were added to the first block. At the second block, homocysteine levels were added to the model. The DIF, DDF, and EOT subscales of the TAS-20 were entered in the third, and last step. The $p$ values $\leq 0.05$ were deemed statistically significant. All statistical testing was two-tailed. Statistical analyses were performed using SPSS for Windows release ver. 10 (SPSS Inc., Chicago, IL, USA). 
Table 1. Results of the linear regression analysis with SSI as dependent variable and other variables as independent

\begin{tabular}{|c|c|c|c|c|c|c|}
\hline & \multicolumn{2}{|c|}{ Unstandardized coefficients } & \multirow{2}{*}{$\frac{\text { Standardized coefficient }}{\text { Beta }}$} & \multirow{2}{*}{$\mathrm{t}$} & \multirow{2}{*}{$p$ value } & \multirow{2}{*}{$\begin{array}{c}95 \% \mathrm{Cl} \\
\text { for } B\end{array}$} \\
\hline & B & SE & & & & \\
\hline Homocysteine levels & 0.39 & 0.08 & 0.42 & 4.01 & $<0.001$ & $0.16-0.46$ \\
\hline DDF & 0.38 & 0.05 & 0.44 & 3.21 & 0.006 & $0.07-0.28$ \\
\hline
\end{tabular}

Only statistically significant variables are shown.

SSI, Scale of Suicide Ideation; SE, standard error; $95 \% \mathrm{CI}, 95 \%$ confidence interval; DDF, difficulty in describing feeling. $\mathrm{R}^{2}=0.64, \mathrm{dF}=55, \mathrm{~F}=9.98, p<0.001$.

\section{RESULTS}

Sex comparisons between all demographic and clinical variables showed no significant differences concerning any of the accounted variables. The mean duration of illness was $15.3 \pm 6.4$ months. The total score for the TAS-20 in the whole sample was $50.3 \pm 13.8$. Twenty-five (37.3\%) patients had a total score $\geq 61$ at the TAS-20, thus being recorded as alexithymic subjects.

Comparison between individuals with or without alexithymia upon controlling for age, gender, smoking status, duration of illness, recurrence and BMI, showed that alexithymic subjects had greater severity of MDD according to the HAM-D $(28.4 \pm 4.2$ vs. $23.5 \pm 3.1$, degree of freedom $[\mathrm{dF}]=1,67 \mathrm{~F}=37.8 p<0.001$, Cohen's $d=1.33$ ), higher homocysteine levels $(15.9 \pm 4.5$ vs. $12.0 \pm 3.2 \mu \mathrm{mol} / \mathrm{L}$, $\mathrm{dF}=1,67 \mathrm{~F}=25.4 p=0.006$, Cohen's $d=1.00)$ and higher SSI scores $(7.1 \pm 2.2$ vs. 2.0 $2.0, \mathrm{dF}=1,67 \mathrm{~F}=94.7 p<$ 0.001 , Cohen's $d=2.43$ ) compared to non-alexithymic cases. Effect size calculation showed that the magnitude of the group effect between alexithymics and nonalexithymics concerning these three variables was large.

In the linear regression models (Table 1) higher homocysteine levels and DDF dimensions of TAS-20 were associated with higher suicide ideation (using SSI as dependent variable). In the current analyses, the $\mathrm{R}^{2}$ values accounted for $64 \%$ of variance in the SSI score. In addition, Durbin-Watson coefficient was 2.004 (near to the optimum of 2.0). A scatter plot of residuals and a plot of regression-standardized residuals indicated a near normal distribution.

\section{DISCUSSION}

To our knowledge, this was the first study that evaluated the relationships between alexithymia, homocysteine levels and suicide ideation in a sample of out- patients with MDD.

Patients with alexithymia showed increased MDD severity, higher homocysteine levels and more severe suicide ideation and these results may be explained according to the Freyberger's concept of acute "secondary alexithymia” as a reaction to stressful situations. ${ }^{13)}$ Acute secondary alexithymia may be explained as a transitory, state-dependent phenomenon that results as an effect of personal distress, and which may decrease once an acute disease episode has resolved. In fact, our results may reflect a state-dependent phenomenon, perhaps related to higher MDD severity. Interestingly, we found that DDF dimension of TAS-20 was associated with higher suicide levels in patients with MDD and this is in line with the findings of a previous study. ${ }^{14)}$ Moreover, these results may be also in accordance with the stress-alexithymia hypothesis ${ }^{15)}$ : patients with alexithymic traits may be suffering from chronic stress reaction that could itself promote persistent and often subclinical increases in inflammatory and oxidative factors such as C-reactive protein, serum lipid dysregulation and homocysteine.

According to Folstein et al., ${ }^{16)}$ high levels of homocysteine would be associated with cerebrovascular disease, monoamine neurotransmitters, and depression of mood itself. According to the later hypothesis, homocysteine levels may cause cerebral vascular disease and neurotransmitter deficiency, which would on turn cause depression of mood and suicide ideation. However, all considered, the results of our study support the notion that depressed alexithymics with DDF may have a homocysteine dysregulation that may be linked in a unclear way to suicide risk. Therefore, we recommend to screen depressed patients for both alexithymia and suicide ideation (as all authors do in their everyday clinical practice), regardless of depression severity, and to evaluate also homocysteine in order to have a further indirect potential biomarker for suicide risk. One can argue that treating hy- 
perhomocysteinemia would reduce suicide risk; however, to date, no studies are present confirming this hypothesis even if oxidative stress may play a role in depression severity thus increasing suicide risk. ${ }^{17,18)}$

To date, it is unclear how hyperhomocysteinemia may contribute to the development and maintenance of psychiatric disorders, alexithymia and suicide ideation, but some studies tried to unravel this issue. ${ }^{19-22)}$ It has been suggested that elevated levels of homocysteine may compromise blood-brain barrier integrity probably increasing its permeability through glutamatergic NMDA receptor-dependent regulation of adherens and tight junctions. $^{23,24)}$ Therefore, hyperhomocysteinemia may cause a direct damage to the neurons causing psychiatric disoders through several mechanisms that may be interconnected $^{25,26)}:$ 1) straight neuronal toxicity through specific receptors on the neurons, inducing cell death, 2) impairing the cerebral vascularization and, particularly, the endothelium of small brain vessels. This may cause neuronal death indirectly also impairing the neurotransmission which is involved in the basic functions of the brain, consequently modifying the regular functioning of the mind of a subject. ${ }^{27)}$ Furthermore, a fault in methylation processes is thought to be essential to the psychiatric manifestations due to the lack of vitamins B6, B12, and folate. ${ }^{28)}$ All these vitamins are closely connected with homocysteine metabolism such that total serum hyperhomocysteine level is considered to be a sensitive and important marker of the functional brain deficit of such vitamins. ${ }^{29,30)}$ Last but not least, as methionine is the precursor of S-adenosylmethionine, which is the direct meth$\mathrm{yl}$ donor in several reactions involved in the biosynthesis of the monoaminergic neurotransmitters, ${ }^{31-33)}$ the altered metabolism of homocysteine and, therefore, the presence of a condition of hyperhomocysteinemia may cause an impairment in the levels of monoamine neurotransmitters causing mood disturbance and suicidal ideation. ${ }^{34)}$ All considered, as the results of our study pointed out that in alexithymic subjects with MDD we found an higher level of homocysteine that was correlated to increased suicide ideation, it can be hypothesized that changes of peripheral blood homocysteine levels may affect brain activity and induce suicidal ideation and alexithymia, but further studies are needed.

The present study was exploratory in nature. As such, we would prompt the following limitations in the inter- pretation of the preliminary results. The first limitation was the relatively small sample size (even if all the evaluated patients were drug naïve). Moreover, even if severity of MDD and suicidal ideation were analysed using clinician-rated rating scales, alexithymia was assessed by a self-rated scale, with possible biases due to the inherent nature of self-rating scales. Furthermore, we employed a cross-sectional design that limits statements regarding causality: our study lacks follow-up data. In fact, the cross-sectional nature of the present study precluded any firm conclusion about any eventual hierarchical role interaction of either alexithymia or hyperomocysteinemia. Finally, in the present study we did not evaluated serum folate levels that may give more meaningful results: future studies should be take into account this point.

Therefore, prospective studies are needed, with repeated-measure design of serum homocysteine prior, during and after the acute pharmacological treatment.

\section{REFERENCES}

1. Otte C, Gold SM, Penninx BW, Pariante CM, Etkin A, Fava M, et al. Major depressive disorder. Nat Rev Dis Primers 2016:2:16065.

2. Li S, Zhang B, Guo Y, Zhang J. The association between alexithymia as assessed by the 20-item Toronto Alexithymia Scale and depression: A meta-analysis. Psychiatry Res 2015;227: 1-9.

3. Honkalampi K, Koivumaa-Honkanen H, Lehto SM, Hintikka J, Haatainen K, Rissanen T, et al. Is alexithymia a risk factor for major depression, personality disorder, or alcohol use disorders? A prospective population-based study. J Psychosom Res 2010;68:269-273.

4. Luminet O, Bagby RM, Taylor GJ. An evaluation of the absolute and relative stability of alexithymia in patients with major depression. Psychother Psychosom 2001;70:254-260.

5. Assies J, Mocking RJ, Lok A, et al. Erythrocyte fatty acid profiles and plasma homocysteine, folate and vitamin B6 and B12 in recurrent depression: Implications for co-morbidity with cardiovascular disease. Psychiatry Res 2015;229:992-998.

6. Ford AH, Flicker L, Singh U, Hirani V, Almeida OP. Homocysteine, depression and cognitive function in older adults. J Affect Disord 2013;151:646-651.

7. American Psychiatric Association. Diagnostic and statistical manual of mental disorders: DSM-IV-TR. 4th ed. Washington, DC:American Psychiatric Association; 2000.

8. First MB, Gibbon M, Spitzer RL, Williams JBW; Department of Biometrics Research, New York State Psychiatric Institute. $S C I D-I$ User's guide for the structured clinical interview for DSM-IV-TR Axis / disorders: SCID 101 for DSM-IV. New York:New York State Psychiatric Institute;2002. 
9. Hamilton M. Development of a rating scale for primary depressive illness. Br J Soc Clin Psychol 1967;6:278-296.

10. Bressi C, Taylor G, Parker J, Bressi S, Brambilla V, Aguglia E, et al. Cross validation of the factor structure of the 20-item Toronto alexithymia scale: an Italian multicenter study. J Psychosom Res 1996;41:551-559.

11. Bagby RM, Taylor GJ, Parker JD. The twenty-item Toronto Alexithymia Scale--Il. Convergent, discriminant, and concurrent validity. J Psychosom Res 1994;38:33-40.

12. Beck AT, Kovacs M, Weissman A. Assessment of suicidal intention: the Scale for Suicide Ideation. J Consult Clin Psychol 1979; 47:343-352.

13. Freyberger H. Supportive psychotherapeutic techniques in primary and secondary alexithymia. Psychother Psychosom 1977;28:337-342.

14. Na KS, Oh SJ, Jung HY, Irene Lee $\mathrm{S}$, Kim YK, Han C, et al. Alexithymia and low cooperativeness are associated with suicide attempts in male military personnel with adjustment disorder: a case-control study. Psychiatry Res 2013;205:220226.

15. Martin JB, Pihl RO. The stress-alexithymia hypothesis: theorectical and empirical considerations. Psychother Psychosom 1985:43:169-176.

16. Folstein M, Liu T, Peter I, Buell J, Arsenault L, Scott T, et al. The homocysteine hypothesis of depression. Am / Psychiatry 2007;164:861-867.

17. Karas Kuželički N. S-adenosyl methionine in the therapy of depression and other psychiatric disorders. Drug Dev Res 2016;77:346-356.

18. Vargas HO, Nunes SO, de Castro MR, Vargas MM, Barbosa DS, Bortolasci CC, et al. Oxidative stress and inflammatory markers are associated with depression and nicotine dependence. Neurosci Lett 2013;544:136-140.

19. Zhou SJ, Zhang LG, Chen HM, Li JY, Li R, Zhang XM, et al. Prevalence and clinical-demographic correlates of hyperhomocysteinemia in inpatients with bipolar disorder in a Han Chinese population. Psychiatry Res 2018;259:364-369.

20. Mabrouk H, Douki W, Mechri A, Younes MK, Omezzine A, Bouslama A, et al. [Hyperhomocysteinemia and schizophrenia: case control study]. Encephale 2011;37:308-313. French.

21. Meier C, Harbrecht U, Liedtke R, Oldenburg J, Conrad R, Imbierowicz K, et al. Relative hyperhomocysteinemia in patients with panic disorder: a case-control study. Neuropsychobiology 2010;62:164-170.

22. Narayan SK, Verman A, Kattimani S, Ananthanarayanan PH, Adithan C. Plasma homocysteine levels in depression and schizophrenia in South Indian Tamilian population. Indian J Psychiatry 2014;56:46-53.

23. Kamath AF, Chauhan AK, Kisucka J, Dole VS, Loscalzo J,
Handy DE, et al. Elevated levels of homocysteine compromise blood-brain barrier integrity in mice. Blood 2006; 107:591-593.

24. Beard RS Jr, Reynolds JJ, Bearden SE. Hyperhomocysteinemia increases permeability of the blood-brain barrier by NMDA receptor-dependent regulation of adherens and tight junctions. Blood 2011;118:2007-2014.

25. Obeid R, McCaddon A, Herrmann W. The role of hyperhomocysteinemia and B-vitamin deficiency in neurological and psychiatric diseases. Clin Chem Lab Med 2007:45:15901606.

26. Herrmann W, Lorenzl S, Obeid R. [Review of the role of hyperhomocysteinemia and B-vitamin deficiency in neurological and psychiatric disorders--current evidence and preliminary recommendations]. Fortschr Neurol Psychiatr 2007;75:515-527. German.

27. Permoda-Osip A, Dorszewska J, Skibinska M, ChlopockaWozniak M, Rybakowski JK. Hyperhomocysteinemia in bipolar depression: clinical and biochemical correlates. Neuropsychobiology 2013;68:193-196.

28. Lippi G, Plebani M. Hyperhomocysteinemia in health and disease: where we are now, and where do we go from here? Clin Chem Lab Med 2012;50:2075-2080.

29. Muntjewerff JW, Kahn RS, Blom HJ, den Heijer M. Homocysteine, methylenetetrahydrofolate reductase and risk of schizophrenia: a meta-analysis. Mol Psychiatry 2006;11: 143-149.

30. Iqbal MP, Lindblad BS, Mehboobali N, Yusuf FA, Khan AH, Iqbal SP. Folic acid and vitamin B6 deficiencies related hyperhomocysteinemia in apparently healthy Pakistani adults; is mass micronutrient supplementation indicated in this population? J Coll Physicians Surg Pak 2009;19:308-312.

31. Taysi S, Keles MS, Gumustekin K, Akyuz M, Boyuk A, Cikman $\mathrm{O}$, et al. Plasma homocysteine and liver tissue $S$-adenosy/methionine, S-adenosy/homocysteine status in vitamin B6-deficient rats. Eur Rev Med Pharmacol Sci 2015;19:154160.

32. De Berardis D, Marini S, Serroni N, Rapini G, lasevoli F, Valchera A, et al. S-adenosyl-L-methionine augmentation in patients with stage II treatment-resistant major depressive disorder: an open label, fixed dose, single-blind study. Scientific WorldJournal 2013;2013:204649.

33. De Berardis D, Orsolini L, Serroni N, Girinelli G, lasevoli F, Tomasetti $\mathrm{C}$, et al. A comprehensive review on the efficacy of $S$-adenosyl-L-methionine in major depressive disorder. CNS Neurol Disord Drug Targets 2016;15:35-44.

34. Walterfang M, Bonnot O, Mocellin R, Velakoulis D. The neuropsychiatry of inborn errors of metabolism. J Inherit Metab Dis 2013;36:687-702. 